Ann. Zootech., I970, 19 (I), 85-87.

NOTE

\title{
UTILISATION DES ALIMENTS CONDENSÉS PAR LES VACHES LAITIERES
}

\author{
M. JOURNET \\ Station de Recherches sur l'Élevage des Ruminants, \\ Centre de Recherches de Clermont-Ferrand, 63-Saint-Genès-Champanelle \\ Institut national de la Recherche agronomique
}

Nous avons montré antérieurement (Journet et JARRIGE, I967) que des vaches laitières alimentées avec un foin de luzerne condensé (broyé et aggloméré) associé à de l'ensilage d'herbe et des betteraves, avaient un niveau d'ingestion élevé et produisaient un lait de composition normale. Au cours de deux nouveaux essais, nous avons étudié l'influence de :

a) l'origine botanique du foin, luzerne ou graminée ;

b) la finesse de broyage du foin ;

c) l'incorporation d'aliments concentrés avec le foin broyé dans un aliment condensé.

Dans l'essai I, nous avons comparé 3 aliments condensés comportant des proportions différentes de foin de luzerne broyé (grille de $3 \mathrm{~mm}$ ) et d'aliment concentré $(0 ; 30 ; 60 \mathrm{p}$. Ioo de céréales + tourteaux); chacun d'eux a été distribué ad libitum à un lot de ro vaches pendant 8 semaines consécutives. Dans l'essai 2, nous avons préparé à partir d'un foin de luzerne et d'un foin de graminées 4 aliments condensés différant par la finesse de broyage (grilles de $3 ; 5$ et Io $\mathrm{mm}$ ) du foin et la proportion d'aliment concentré (0; 30 et 50 p. 10o). Ils ont été distribués ad libitum à 4 Iots de 3 vaches selon un dispositif en carré latin ; les périodes expérimentales duraient 4 semaines. Dans les 2 essais, avant et après l'expérience, les vaches ont été alimentées avec un régime cle composition normale. Pendant l'expérience, en plus de l'aliment condensé, elles ont reçu un aliment concentré en farine ( $96 \mathrm{p}$. roo de céréales $+4 \mathrm{p}$. Ioo de minéraux) pour couvrir leurs besoins, lorsque c'était nécessaire. Elles disposaient d'une abondante litière de paille. Les quantités ingérées, la production de lait et le taux butyreux (Gerber) ont été mesurés individuellement chaque jour et le taux de protéines du lait (noir amido) une fois par semaine.

Les quantités d'aliments condensés ingérées sont élevées dans les 2 essais (tabl. I). La quantité de matière sèche totale ingérée (en moyenne $3,2 \mathrm{p}$. Ioo du poids vif) est supérieure de près de 20 p. Ioo à celles des rations classiques; elle est cependant plus faible dans le premier essai en raison semble-t-il de la dureté des aliments condensés; elle est peu influencée par la finesse de broyage ; elle est plus faible avec le foin de graminée qu'avec le foin de luzerne et lorsque la proportion d'aliment concentré dépasse $3 \circ$ p. $100(p<0,05)$.

La composition du lait a été modifiée : par rapport aux régimes classiques distribués au cours des périodes pré- et post-expérimentales, le taux butyreux a diminué de I, 5 à $15,5 \mathrm{~g}$ P. I ooo et le 
taux de protéines a augmenté de $\mathrm{I}, \mathrm{o}$ à $3,2 \mathrm{~g} \mathrm{p}$. I ooo selon les régimes (tabl. I). Des 3 facteurs étudiés seule la proportion d'aliment concentré a eu un effet; lorsqu'elle ne dépasse pas $30 \mathrm{p}$. Ioo l'effet dépressif sur le taux butyreux reste faible et indépendant de la finesse de broyage du foin.

TABLEAU I

Quantités ingérées, production et composition du lait

\begin{tabular}{|c|c|c|c|c|c|c|c|c|c|}
\hline \multirow{2}{*}{ Essai } & \multirow{2}{*}{\multicolumn{2}{|c|}{$\begin{array}{l}\text { Dénomination } \\
\text { de l'aliment }\left({ }^{1}\right)\end{array}$}} & \multicolumn{2}{|c|}{$\begin{array}{l}\text { Quantités ingérces } \\
\text { (M.S. p. } 100 \text { P.V.) }\end{array}$} & \multirow{2}{*}{$\begin{array}{l}\text { I.ait } \\
(\mathrm{kg})\end{array}$} & \multicolumn{2}{|c|}{$\begin{array}{l}\text { Teneur du lait } \\
\text { (g p. } 1000)\end{array}$} & \multicolumn{2}{|c|}{\begin{tabular}{l}
\multicolumn{2}{c}{ Variation } \\
$\left(\mathrm{g}\right.$ p. $\left.1000\left({ }^{2}\right)\right)$
\end{tabular}} \\
\hline & & & $\begin{array}{l}\text { Aliment } \\
\text { condensé }\end{array}$ & $\begin{array}{l}\text { Ration } \\
\text { totale }\end{array}$ & & $\begin{array}{l}\text { Matières } \\
\text { grasses }\end{array}$ & $\begin{array}{l}\text { Matières } \\
\text { azotées }\end{array}$ & $\begin{array}{l}\text { Matières } \\
\text { grasses }\end{array}$ & $\begin{array}{l}\text { Matières } \\
\text { azotées }\end{array}$ \\
\hline \multirow{3}{*}{ I } & Luzerne & $3-0$ & 2,22 & 2,83 & 14,0 & 32,0 & 33,7 & $-\quad 4,8$ & $\cdots \quad 1,9$ \\
\hline & Luzerne & $3-30$ & 2,83 & 2,89 & 13,9 & 33,6 & 33,6 & $-\quad 3,9$ & $+2,5$ \\
\hline & Luzerne & $3-60$ & 2,71 & 2,72 & $1 /, 1$ & 22,8 & 34,8 & $-15,5$ & $+3,1$ \\
\hline \multirow{8}{*}{ II } & Luzerne & $3-0$ & 2,43 & 3,62 & 17,2 & $3^{\prime}, 9$ & $3 t, 3$ & $-\quad 2,3$ & $+1,0$ \\
\hline & I.uzerne & $5-10$ & 2,61 & 3,68 & 17,11 & 37,8 & $3 \mathbf{y}^{\prime} \mathbf{t}$ & $-\quad 2,3$ & $\therefore \quad 1, \geq$ \\
\hline & Luzerne & $10-30$ & 3,26 & 3,48 & 16,7 & $3 ', 7$ & 33,6 & $-\quad 2,3$ & $\ldots \quad 0,7$ \\
\hline & Luzerne & $10-50$ & 3,13 & 3,28 & 16,9 & 30,7 & 35,0 & $-6, \tilde{5}$ & $\therefore \quad 1,6$ \\
\hline & Graminées & $3-0$ & $-2,53$ & 3,21 & 15,5 & 34,2 & 34,1 & $-\quad 2,5$ & $\therefore \quad 1,4$ \\
\hline & Graminées & $5-10 \mid$ & 2,72 & 3,27 & 15,1 & 34,9 & 34,5 & $-1,8$ & -1 1,3 \\
\hline & Graminées & $10-30$ & 3,16 & 3,31 & 16,0 & 35,3 & 35,9 & $-5,8$ & $\div 3,11$ \\
\hline & Graminées & $10-i j 0$ & $-2,88$ & 3,08 & 16,5 & 31,1 & 35,9 & $\longrightarrow 5,8$ & $+3,2$ \\
\hline
\end{tabular}

(1) Le premier chiffre indique en mm le diamètre des mailles du broyeur, le deuxième la proportion d'aliment concentré dans l'aliment condensé. Luzerne : foin de luzerne; Graminées : foin de graminées.

${ }^{(2)}$ Différences calculées par intrapolation par rapport aux périodes pré- et post-expérimentales effectuées sur régime normal.

Avec 50 p. roo d'aliment concentré l'effet dépressif devient plus marqué malgré un broyage grossier du foin ; c'est avec $60 \mathrm{p}$. Ioo d'aliment concentré et un broyage fin qu'il est maximum.

Inversement le taux de matières azotées augmente mais en raison surtout de la suralimentation énergétique $(r=+0,78$ avec la différence : apports - besoins, exprimée en unités fourragères pour les I I régimes étudiés). Il augmente cependant avec les aliments condensés ne contenant pas d'aliment concentré sans que le niveau des apports énergétiques s'accroisse.

La production de matières grasses diminue très rapidement (de moitié en 2 mois) dans l'essai I avec l'aliment contenant $60 \mathrm{p}$. Ioo d'aliment concentré, bien que l'apport énergétique (UF) soit de 17 et 38 p. Ioo plus élevé qu'avec les 2 autres aliments condensés. En contrepartie ces vaches ont un gain de poids vif de $360 \mathrm{~g}$ par jour alors que celle des autres lots restent à poids constant.

Ces essais montrent que des rations constituées presque entièrement par des aliments condensés occasionnent, même avec un broyage grossier du foin et la présence de paille dans la litière, des baisses du taux butyreux. Pour éviter ces baisses du taux butyreux et de la production de matières grasses et un engraissement des animaux, il est nécessaire de broyer le foin avec des grilles dont le diamètre des mailles est supérieur à $5 \mathrm{~mm}$ et, surtout, de ne pas incorporer de céréales et de tourteaux dans l'aliment condensé à un taux supérieur à zo p. roo.

Ces résultats sont en accord avec la plupart des travaux rapportés par Minson (1962) et L. A., MOORE (1964). 


\section{SUMMARY}

\section{USE OF PELI,ETED FEEDS FOR DAIRY COWS}

Th: ingestion rate is high, but variable ( 2.7 to $3.7 \mathrm{~kg}$ of dry matter for too $\mathrm{kg}$ of live weight), for alfalfa or grass hay, with or without a concentrated feed incorporated. If the hay is not ground too fine ( 3 or 5 or $10 \mathrm{~mm}$ screens), and if the proportion of concentrated feed is not higher than $3^{\circ} \mathrm{p}$. Ioo, the fat content only decreases from 2 to $5 \mathrm{~g}$ p. I ooo. With a higher proportion of concentrated feed, it diminishes considerably (from 6 to I $5 \mathrm{~g}$ p. I ooo), milk fat rapidly decreases, and the animals gain weight.

\section{RÉFÉRENCES BIBLIOGRAPHIQUES}

Journet M., Jarrige R., I967. Utilisation des aliments broyés et agglomérés par les bovins. II. Utilisation comparée par la vache laitière du foin de luzerne condensé et du foin de luzerne normal associés à de l'ensilage et des betteraves. Ann. Zootech., 16, 27I-289.

IIISSON D. J., I962. The effect of grinding, pelleting and wafering on the fecding value of roughages : a review - Contrib. ${ }^{\circ} 84$, Anim. Res. Institute, Canada Dep. Agric., Ottawa, Ontario,

IIOORE L. A., I964. Symposium on forage utilization : Nutritive value as affected by physical form. 1. General principles involved with ruminants and effect of feeding pelleted or wafered forage to diary attle. Anim. Sci., 32, $230-238$. 